\title{
Nodal failure patterns and utility of elective nodal irradiation in submandibular gland carcinoma treated with postoperative radiotherapy - a multicenter experience
}

Cheng-En Hsieh 1,7,11,12, Li-Yu Lee 2,7, Yung-Chih Chou', Kang-Hsing Fan 1,7, Ngan-Ming Tsang 1,7,9, Joseph Tung-Chieh Chang ${ }^{1,7,10}$, Hung-Ming Wang ${ }^{3,7}$, Shu-Hang Ng ${ }^{4,7}$, Chun-Ta Liao ${ }^{5,7}$, Tzu-Chen Yen ${ }^{6,7}$, Ku-Hao Fang ${ }^{5,7,8}$ and Chien-Yu Lin ${ }^{1,7,13^{*}}$ (D)

\begin{abstract}
Background: The patterns of nodal relapse in submandibular gland carcinoma (SMGC) patients treated with postoperative radiotherapy (PORT) remain unclear. This study aims to investigate the nodal failure patterns and the utility of elective nodal irradiation (ENI) in SMGC patients undergoing PORT.

Methods: We retrospectively enrolled 65 SMGC patients who underwent PORT between 2000 and 2014. The nodal failure sites in relation to irradiation fields and pathological parameters were analyzed. ENI regions were categorized into three bilateral echelons (first, levels I-II; second, level III; and third, levels IV-V). Extended ENI was defined as coverage of at least the immediately adjacent uninvolved echelons bilaterally; otherwise, limited ENI was administered.
\end{abstract}

Results: Thirty patients (46\%) were stage III-IV, and 18 (28\%) were pN+. Neck irradiation included limited (72\%) and extended ENI (28\%). With a median follow-up of 79 months, 11 patients (17\%) developed nodal failures (ipsilateral, $N=6$; contralateral, $N=7), 7$ (64\%) of whom relapsed in the adjacent uninvolved echelons. We identified pN+ $(P=0.030)$, extranodal extension ( $E N E, P=0.002)$, pT3-4 $(P=0.021)$, and lymphovascular invasion $(L V I, P=0.004)$ as significant predictors of contralateral neck recurrence. Extended ENI significantly improved regional control (RC) in patients with $\mathrm{pN}+(P=0.003)$, ENE $(P=0.022)$, pT3-4 $(P=0.044)$, and LVI $(P=0.014)$, and improved disease-free survival (DFS) in patients with $\mathrm{pN}+(P=0.034)$. For patients with $\geq 2$ coincident adverse factors, extended ENI significantly increased RC $(P<0.001)$, distant metastasis-free survival $(P=0.019)$, and DFS $(P=0.007)$; conversely, no nodal recurrence was observed in patients without these adverse factors, even when only the involved echelon was irradiated.

Conclusions: Nodal failure is not uncommon in SMGC patients treated with PORT if pN+, ENE, pT3-4, and LVI are present. Extended ENI should be considered, particularly in patients with multiple pathological adverse factors.

Keywords: Submandibular gland cancer, Postoperative radiotherapy, Elective nodal irradiation, Nodal failure pattern

\footnotetext{
* Correspondence: qqvirus1022@gmail.com

${ }^{1}$ Radiation Oncology, Chang Gung Memorial Hospital at Linkou and Chang

Gung University, No. 5, Fuxing St., Guishan Dist, Taoyuan City 33305, Taiwan,

Republic of China

${ }^{7}$ Head and Neck Oncology Group, Chang Gung Memorial Hospital at Linkou

and Chang Gung University, Taoyuan City, Taiwan, Republic of China

Full list of author information is available at the end of the article
}

(c) The Author(s). 2018 Open Access This article is distributed under the terms of the Creative Commons Attribution 4.0 International License (http://creativecommons.org/licenses/by/4.0/), which permits unrestricted use, distribution, and

reproduction in any medium, provided you give appropriate credit to the original author(s) and the source, provide a link to the Creative Commons license, and indicate if changes were made. The Creative Commons Public Domain Dedication waiver (http://creativecommons.org/publicdomain/zero/1.0/) applies to the data made available in this article, unless otherwise stated. 


\section{Background}

Submandibular gland carcinomas (SMGCs) are rare malignancies, accounting for less than $10 \%$ of salivary gland neoplasms and $1 \%$ of head and neck cancers [1-3]. En bloc radical resection is the mainstay of treatment [4], and postoperative radiotherapy (PORT) has been utilized in patients with adverse pathological factors including advanced stage, high-grade tumors, positive surgical margins, bone invasion, and perineural invasion (PNI) [5-9]. The behavior of SMGC and the optimal PORT treatment remain unclear. Data on combined-modality treatments usually encompass parotid gland cancer patients, and the nodal failure patterns and clinical utility of elective nodal irradiation (ENI) for SMGC remain vague $[6,10-13]$. The currently recommended nodal irradiation fields are based on the treatment experience of different salivary gland malignancies (most of them arising from the parotid gland).

The incidence of neck metastases in parotid gland carcinoma is reportedly $12-25 \%[6,8,11,14,15]$, with no risk to the contralateral neck $[11,16]$. However, the submandibular gland has more extensive lymphatic drainage; hence, SMGC is more amenable to nodal metastasis [6, 10, 17]. According to a detailed study by the Dutch Head and Neck Oncology Group, pathologically positive nodes were detected in $42 \%$ of SMGC patients, and the nodal metastasis rate approached $60 \%$ for high-grade and advanced T-stage tumors [6]. Even when a unique histology entity (adenoid cystic carcinoma, ACC) is considered, the rates of nodal metastases from submandibular gland malignancies are higher than those observed for parotid gland tumors $(22.5 \%$ vs $14.5 \%$, respectively) [18]. Furthermore, increased risks of contralateral neck metastasis (12.4-fold) and occult nodal metastasis have been reported in oral cavity squamous cell carcinoma that directly invades into the floor of the mouth [19-21]. As the submandibular glands are proximal to the floor of the mouth and midline of the neck, it is unclear whether contralateral neck treatment should be omitted as it is for parotid gland cancer patients. Therefore, we conducted this retrospective multicenter study to review the long-term outcomes of SMGC patients treated with radical resection and PORT. Additionally, we investigated the use and treatment outcomes of ENI, and identified patients who would most benefit from extended ENI.

\section{Methods}

\section{Patients and clinical workup}

A total number of 74 patients with SMGC treated with radical surgery and PORT at Linkou, Kaohsiung, Keelung, and Chiayi Chang Gung Memorial Hospitals between January 2000 and December 2014 were identified. Patients with distant metastasis at diagnosis $(N=5)$, history of prior irradiation $(N=3)$, and lymphoma histology $(N=1)$ were excluded; 65 patients were ultimately enrolled. The staging workups and follow-up schedules were described previously [22]. The cancer staging was revised according to the seventh edition (2010) of the American Joint Committee on Cancer staging criteria, and the tumor histology was defined according to 2005 World Health Organization classification.

\section{Treatment}

All subjects were treated with radical submandibulectomy, and neck dissection was performed for those with clinical nodal involvement or locally advanced tumors. PORT was administered using megavoltage photon irradiation, 1.8-2 Gy per fraction, five times per week using either three-dimensional conformal radiotherapy, intensity-modulated radiation therapy, or volumetric modulated arc therapy delivery systems. The prophylactic dose of ENI was 46-50 Gy with a 60-66 Gy boost to high-risk regions. ENI was generally administered to patients presenting with adverse pathological features. The neck irradiation regions were categorized into 3 consecutive bilateral echelons according to the lymphatic drainage anatomy of the submandibular gland: the first echelon was for levels I-II, the second for level III, and the third for levels IV-V (Fig. 1a, b). Extended ENI encompassed the adjacent uninvolved echelons bilaterally according to the pathological tumor extension (Fig. 1c). Otherwise, limited ENI, defined as the irradiation of the involved echelon alone or ipsilateral ENI for adjacent uninvolved echelons without prophylactic contralateral neck treatment, was administered. Concurrent chemotherapy was generally applied for patients with adverse pathological features [23]; intravenous cisplatin was the most commonly used agent (typically at $100 \mathrm{mg} / \mathrm{m}^{2}$ once every 3 weeks or $40 \mathrm{mg} / \mathrm{m}^{2}$ once per week) [24, 25].

\section{Statistical analysis}

Intergroup differences in continuous variables were tested using independent Student's $t$-tests. Categorical data were compared using the Pearson's chi-squared or the Fisher's exact test, as appropriate. Local control (LC), regional control (RC), distant metastasis-free survival (DMFS), disease-free survival (DFS), and overall survival (OS) were calculated from the date of surgery to the date of detection of the relevant events, respectively. Survival curves were plotted using the Kaplan-Meier method and compared using the log-rank test. Multivariate analysis was performed using Cox proportional hazards regression models with a stepwise forward conditional manner. Variables were retained in the model if their significance levels were $<0.05$. Results are expressed as hazard ratios with their 95\% confidence intervals. All data analyses were performed using the SPSS 20.0 statistical software (IBM Corporation, Armonk, New York, USA). 


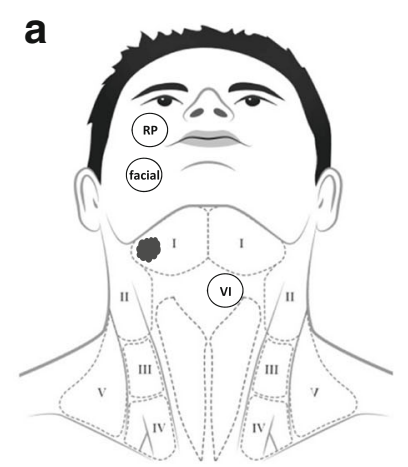

Nodal Levels

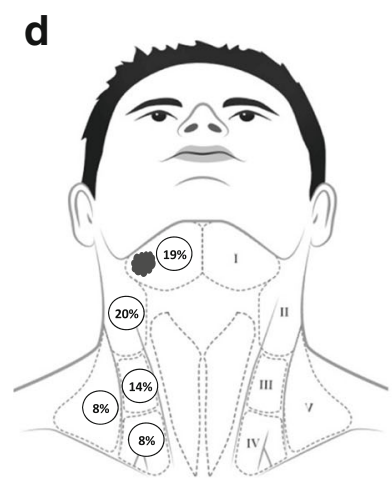

Pathological nodal metastasis rates

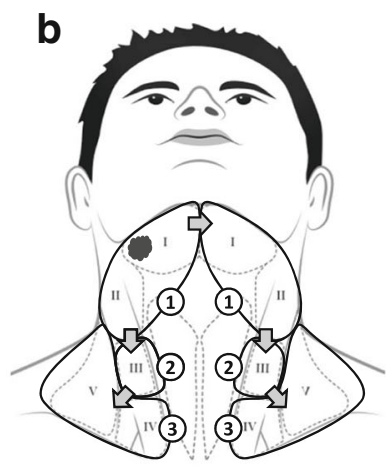

Anatomic grouping of neck echelons

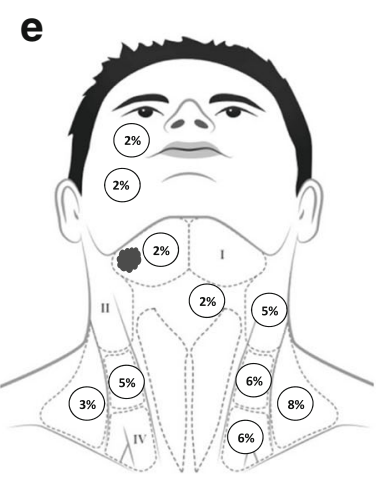

Nodal recurrence rates

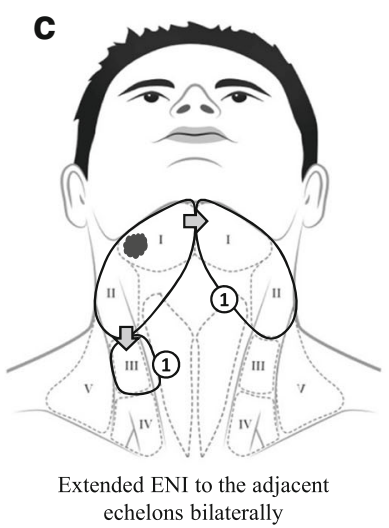

$\mathbf{f}$

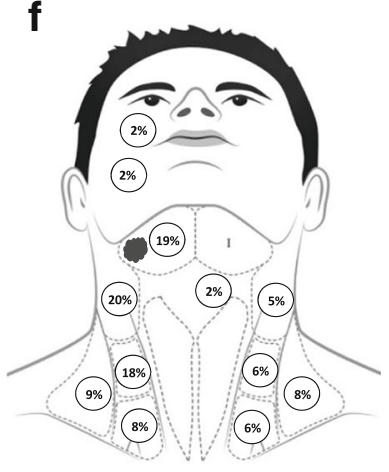

Total nodal metastatic rates

Fig. 1 a Depiction of each of Level I-V, retropharyngeal (RP) and facial nodes. b The neck irradiation regions were categorized into 3 consecutive bilateral echelons according to lymphatic drainage: the first echelon for levels I-II, the second for level III, and the third for levels IV-V. c Taking a submandibular gland carcinoma (SMGC) patient with multiple risk factors as an example, if the tumor invades level I, extended elective nodal irradiation (ENI) of the adjacent bilateral echelons involves the ipsilateral levels I-III and contralateral levels I-II. $\mathbf{d}$ Pathological nodal metastatic rates in 24 patients who received ipsilateral neck dissection. e Nodal recurrent rates and (F) total nodal metastatic rates in 65 SMGC patients treated with surgery and postoperative radiotherapy

\section{Results}

\section{Patient characteristics}

Patient characteristics are demonstrated in Table 1. The median age was 53 years, and the most common histological subtypes were ACC, carcinoma ex pleomorphic adenoma, and mucoepidermoid carcinoma.

Clinically positive nodes were observed in 15 patients (23\%); 24 patients (37\%) received ipsilateral neck dissection while none received contralateral neck surgery. Consequently, pathological nodal metastasis was detected in 18 patients (28\%). The distribution of pathological positive nodes is illustrated in Fig. 1d.

\section{Adjuvant treatments}

The median cumulative radiation dose was $66 \mathrm{~Gy}$, and only one patient received a dose less than 50 Gy due to poor health. The median time from surgery to PORT initiation was 34 (range, 11-71) days; the median time was 35 (range, 11-71) days and 29 (range, 14-49) days among those with limited ENI and extended ENI, respectively. Concurrent chemoradiotherapy was administered to 24 patients (limited ENI, $N=16$; extended ENI, $N=8$ ), the most commonly used regimen was intravenous cisplatinbased $(N=23)$. Single-agent cisplatin was administered to 18 patients, whereas concurrent oral uracil-tegafur and cisplatin were administered to the remaining 5 patients. The median cumulative cisplatin dose was $200 \mathrm{mg} / \mathrm{m}^{2}$. Sixteen of the 23 patients (70\%) completed their planned chemotherapy course; one patient received concurrent cetuximab treatment. Neither neoadjuvant nor adjuvant chemotherapy was administered.

\section{Neck irradiation fields}

Extended ENI was performed in 18 patients (28\%), covering the next adjacent uninvolved echelons bilaterally $(N=8)$ or $\geq 2$ additional echelons $(N=10)$. Otherwise, limited ENI was performed in the remaining 47 patients; the irradiated neck regions consisted of involved echelons alone in 30 patients (46\%) and of ipsilateral adjacent uninvolved echelons in 17 (26\%). Notably, 12 of these patients received unintentional irradiation doses to the 
Table 1 Patient characteristics

\begin{tabular}{|c|c|c|}
\hline Characteristic & $N$ & $\%$ \\
\hline \multicolumn{3}{|l|}{ Sex } \\
\hline Female/Male & $35 / 30$ & $54 / 46$ \\
\hline \multicolumn{3}{|l|}{ Age (years) } \\
\hline Median (range) & $53(24-79)$ & \\
\hline \multicolumn{3}{|l|}{ Performance score } \\
\hline ECOG 0-1 & 65 & 100 \\
\hline \multicolumn{3}{|l|}{ T stage } \\
\hline pT1 & 15 & 23 \\
\hline pT2 & 24 & 37 \\
\hline pT3 & 20 & 31 \\
\hline pT4a & 6 & 9 \\
\hline \multicolumn{3}{|l|}{ Tumor size (cm) } \\
\hline Median (range) & $2.9(0.5-8.0)$ & \\
\hline \multicolumn{3}{|l|}{ N stage } \\
\hline pNO & 6 & 9 \\
\hline pN1 & 3 & 5 \\
\hline $\mathrm{pN} 2 \mathrm{~b}$ & 15 & 23 \\
\hline $\mathrm{cN} / \mathrm{pN} \mathrm{x}^{\mathrm{a}}$ & 41 & 63 \\
\hline \multicolumn{3}{|l|}{ Disease stage } \\
\hline I & 13 & 20 \\
\hline$\|$ & 22 & 34 \\
\hline III & 11 & 17 \\
\hline IVa & 19 & 29 \\
\hline \multicolumn{3}{|l|}{ Staging modality } \\
\hline$C T$ & 49 & 75 \\
\hline MRI & 6 & 9 \\
\hline${ }^{18}$ F-FDG-PET & 23 & 35 \\
\hline \multicolumn{3}{|l|}{ Surgical margin } \\
\hline$<1 \mathrm{~mm} / \geq 1 \mathrm{~mm}$ & $46 / 19$ & $71 / 29$ \\
\hline \multicolumn{3}{|l|}{ Histology } \\
\hline Adenoid cystic carcinoma & 29 & 45 \\
\hline Carcinoma ex pleomorphic adenoma & 11 & 17 \\
\hline Mucoepidermoid carcinoma & 8 & 12 \\
\hline Lymphoepithelial carcinoma & 6 & 9 \\
\hline Squamous cell carcinoma & 4 & 6 \\
\hline Salivary duct carcinoma & 4 & 6 \\
\hline Adenocarcinoma & 2 & 3 \\
\hline Myoepithelial carcinoma & 1 & 2 \\
\hline \multicolumn{3}{|l|}{ Histology grading } \\
\hline Low to intermediate/High & $6 / 59$ & 9/91 \\
\hline \multicolumn{3}{|l|}{ Pathological features } \\
\hline Perineural invasion & 35 & 54 \\
\hline Extranodal extension & 12 & 19 \\
\hline Bone invasion & 2 & 3 \\
\hline
\end{tabular}

Table 1 Patient characteristics (Continued)

\begin{tabular}{lll}
\hline Characteristic & $N$ & $\%$ \\
\hline Skin invasion & 1 & 2 \\
$\begin{array}{l}\text { Lymphovascular invasion } \\
\text { Neck dissection }\end{array}$ & 18 & 28 \\
None & 41 & 63 \\
Ipsilateral elective & 9 & 14 \\
Ipsilateral therapeutic & 15 & 23 \\
Contralateral & 0 & 0 \\
$<18$ nodes & 22 & 34 \\
$\geq 18$ nodes & 17 & 26
\end{tabular}

Nodal irradiation

$\begin{array}{lll}\text { Extended ENI } & 18 & 28 \\ \text { Limited ENI } & 47 & 72 \\ \text { Concurrent chemotherapy } & 24 & 37 \\ \text { Radiotherapy technique } & & \\ \text { 3D-CRT } & 19 & 29 \\ \text { IMRT } & 31 & 48 \\ \text { VMAT } & 15 & 23\end{array}$

Radiotherapy dose (Gy)

Median (range)

$66(32-72)$

Abbreviations: 3D-CRT three-dimensional conformal radiotherapy, $C T$ computed tomography, ECOG Eastern Cooperative Oncology Group, ENI elective nodal irradiation, ${ }^{18}$ F-FDG-PET 18 F-fluorodeoxyglucose positron emission tomography, IMRT intensity-modulated radiation therapy, MRI magnetic resonance imaging, VMAT volumetric modulated arc therapy ${ }^{a}$,no elective neck dissection in cNO patients

contralateral levels I $(N=7)$ or I/II $(N=5)$ that encountered the radiation beam paths.

\section{Treatment outcomes and nodal failure patterns}

The median follow-up time for the survivors was 79 (range, $19-183)$ months. By the end of the study, 21 patients (32\%) had died owing to cancer recurrence in 16 and intercurrent diseases in five (coronary artery disease, $N=2$; pneumonia, $N=2$; and cerebral hemorrhage, $N=1$ ). Three patients developed secondary malignancies (gum squamous cell carcinoma, ovarian adenocarcinoma, and cholangiocarcinoma, respectively); the patient with secondary cholangiocarcinoma died of uncontrolled tumor bleeding resulting from SMGC locoregional recurrence (patient \#11), and the two other patients were alive with controlled secondary malignancies. The five- and 10-year OS rates were 72 and $63 \%$ for the entire cohort, respectively.

Cancer recurrence was recorded in 21 patients (32\%). The five- and 10-year DFS rates were 64 and 64\%, respectively. The predominant form of treatment failure was distant metastasis (20 patients), and the five- and 10-year DMFS rates were both $66 \%$. Three patients had local recurrence; among them, perineural tumor recurrence was 
recorded in two ACC patients who were treated with surgical bed irradiation alone (hypoglossal nerve, $N=1$, \#11; facial and lingual nerves, $N=1$, \#12; Table 2, Additional file 1: Figure S1). When we investigated the highest margin of the irradiation fields, we identified that coverage up to the transverse process of the first cervical vertebrae (for level II neck coverage only), mastoid tip, and skull base were performed in 10 (35\%), 1 (3\%), and 18 (62\%) patients while 2 (20\%), $0(0 \%)$, and $0(0 \%)$ had outfield recurrence $(P=0.180)$. The five- and 10 -year LC rates 96 and $92 \%$, respectively.

Eleven patients (17\%) experienced neck recurrence, with five- and 10 -year RC rates of $80 \%$ and $80 \%$, respectively. The median time to nodal recurrence was 25 (range, 6-57) months in 10 patients with limited ENI and 52 months in 1 patients with extended ENI. Ipsilateral and contralateral neck failures were documented in six and seven patients, respectively; the distributions of recurrent nodes and total nodal metastatic rates are shown in Fig. 1e-f. Seven patients (64\%) developed nodal recurrence in the adjacent uninvolved echelons. The detailed neck nodal failure sites and corresponding radiation fields are shown in Table 2. Ten of the 11 patients who experienced nodal relapse also developed uncontrolled distant metastases.

\section{Univariate and multivariate analyses of adverse pathological factors}

On univariate analysis (Table 3), the presence of $\mathrm{pN}+$, ENE, pT3-4, LVI, and PNI was significantly correlated with inferior RC, LC, DMFS, and DFS. A significantly worse five-year LC rate was observed in patients with $\mathrm{pN}+$. The presence of $\mathrm{pN}+$, ENE, pT3-4, and LVI was each a significant predictor of poorer OS.

Multivariate analyses revealed that pT3-4 and LVI significantly correlated with poorer RC. No statistically significant prognosticator for LC was identified (Table 3). Notably, pT3-4, LVI, and PNI were identified as significant independent predictors of DMFS, whereas $\mathrm{pN}+$, PNI, and $\mathrm{pT} 3-4$ were significantly correlated with worse DFS; $\mathrm{pN}+$ and $\mathrm{pT} 3-4$ were significant predictors of inferior OS.

\section{Predictors of nodal recurrence}

Neither univariate nor multivariate analyses revealed significant predictors of ipsilateral nodal recurrence; only a trend toward worse ipsilateral nodal failure was observed in patients with pN+ or pT3-4 (Table 4). However, pN+, ENE, pT3-4, and LVI were significant predictors of contralateral neck recurrence on univariate analysis; whereas ENE and LVI were significant independent prognosticators of the same on multivariate analysis.

Table 2 Neck irradiation fields and failure patterns in 12 submandibular gland carcinoma patients who developed locoregional recurrence after postoperative radiotherapy

\begin{tabular}{|c|c|c|c|c|c|c|c|c|c|c|c|c|c|c|c|c|c|c|c|c|c|c|c|}
\hline \multirow[b]{2}{*}{ Case } & \multirow[b]{2}{*}{ Histology } & \multirow[b]{2}{*}{ pT } & \multirow[b]{2}{*}{$\mathrm{N}$ stage } & \multirow[b]{2}{*}{ Stage } & \multirow[b]{2}{*}{ ENE } & \multirow[b]{2}{*}{ PNI } & \multirow[b]{2}{*}{ LVI } & \multirow[b]{2}{*}{ M } & \multirow[t]{2}{*}{ Local } & \multicolumn{7}{|c|}{ Ipsilateral neck } & \multicolumn{7}{|c|}{ Contralateral neck } \\
\hline & & & & & & & & & & I & II & III & IV & VA & VB & Others & I & II & III & IV & VA & VB & Others \\
\hline 1 & CXPA & 3 & $\mathrm{pN} 2 \mathrm{~b}$ & IVa & + & + & + & + & & $\mathrm{N}$ & $\mathrm{N}$ & $\mathrm{N}$ & $\mathrm{N}$ & $\mathrm{N}$ & & & & $\mathrm{F}$ & $\mathrm{F}$ & $\mathrm{F}$ & $\mathrm{F}$ & $\mathrm{F}$ & \\
\hline 2 & CXPA & 3 & pN0 & III & - & - & + & - & & & & & & & & & & & & $\mathrm{F}$ & & $\mathrm{F}$ & \\
\hline 3 & SDC & 3 & $\mathrm{pN} 2 \mathrm{~b}$ & IVa & + & + & + & - & & $\mathrm{N}$ & $\mathrm{N}$ & & & NF & $\mathrm{F}$ & $\mathrm{RP}$ & & & & & $\mathrm{F}$ & $\mathrm{F}$ & VI \\
\hline 4 & CXPA & 2 & $\mathrm{cN} 0 / \mathrm{pNx}^{\dagger} \dagger$ & II & - & + & + & + & & & & & & & $\mathrm{F}$ & & & & $\mathrm{F}$ & $\mathrm{F}$ & & $\mathrm{F}$ & \\
\hline 5 & $\mathrm{SCC}$ & $4 a$ & $\mathrm{pN} 2 \mathrm{~b}$ & IVa & + & + & + & - & $\mathrm{F}$ & & $\mathrm{N}$ & & & & & & & $\mathrm{F}$ & $\mathrm{F}$ & & & & \\
\hline 6 & CXPA & 3 & $\mathrm{pN} 2 \mathrm{~b}$ & IVa & + & + & + & + & & & $\mathrm{N}$ & $\mathrm{N}$ & & & & & & $\mathrm{F}$ & & & & & \\
\hline 7 & $\mathrm{ACC}$ & 3 & pN0 & III & - & + & - & + & & & & & & & & & & & $\mathrm{F}$ & $\mathrm{F}$ & & $\mathrm{F}$ & \\
\hline 8 & $\mathrm{ACC}$ & 3 & $\mathrm{pN} 1$ & III & - & + & + & + & & & $\mathrm{N}$ & & & & & facial & & & & & & & \\
\hline 9 & $\mathrm{ACC}$ & $4 a$ & pN0 & IVa & - & + & - & + & & & & $\mathrm{F}$ & & & & & & & & & & & \\
\hline 10 & $\mathrm{ACC}$ & $4 a$ & $\mathrm{pN} 0$ & IVa & - & + & - & + & & & & $\mathrm{F}$ & & & & & & & & & & & \\
\hline 11 & $\mathrm{ACC}$ & 1 & $\mathrm{pN} 2 \mathrm{~b}$ & IVa & - & + & - & + & $\mathrm{F}^{*}$ & NF & & $\mathrm{F}$ & & & & & & & & & & & \\
\hline 12 & $\mathrm{ACC}$ & 3 & pN0 & III & - & + & - & + & $\mathrm{F} \#$ & & & & & & & & & & & & & & \\
\hline
\end{tabular}

Gray screentones: areas of neck irradiation. Frames: areas of nodal dissection

Abbreviations: ACC adenoid cystic carcinoma, ENE extranodal extension, CXPA carcinoma ex pleomorphic adenoma, $F$ site of failure, LVI lymphovascular invasion, $M$ surgical margins, $N$ pathologically involved nodal region, $P N I$ perineurial invasion, $P T$ pathological T stage, $R P$ retropharyngeal nodal failure, $S C C$ squamous cell carcinoma, SDC salivary duct carcinoma

†, no elective neck dissection in cN0 patients; Perineural tumor recurrence at *hypoglossal nerve (Additional file 1: Figure S1B) and \#facial and lingual nerves (Additional file 1: Figure S1A). 
Table 3 Univariate and multivariate analyses of adverse pathological factors

\begin{tabular}{|c|c|c|c|c|c|c|c|c|c|c|c|}
\hline \multicolumn{2}{|l|}{ Univariate analysis } & \multicolumn{2}{|l|}{$\mathrm{RC}(\%)$} & \multicolumn{2}{|l|}{ LC (\%) } & \multicolumn{2}{|c|}{ DMFS (\%) } & \multicolumn{2}{|l|}{ DFS (\%) } & \multicolumn{2}{|l|}{ OS (\%) } \\
\hline Variable & $N$ & 5-year & $P$ & 5-year & $P$ & 5-year & $P$ & 5-year & $P$ & 5-year & $P$ \\
\hline \multicolumn{12}{|l|}{ N stage } \\
\hline $\mathrm{pN}+$ & 18 & 49.6 & 0.002 & 75.0 & 0.015 & 31.1 & $<0.001$ & 24.5 & $<0.001$ & 37.5 & $<0.001$ \\
\hline $\mathrm{pNO} \& \mathrm{cNO} / \mathrm{pNx} x^{\mathrm{a}}$ & 47 & 87.7 & & 100 & & 77.5 & & 77.8 & & 85.2 & \\
\hline \multicolumn{12}{|l|}{ Extranodal extension } \\
\hline Yes & 12 & 55.4 & 0.004 & 83.3 & 0.149 & 21.2 & $<0.001$ & 21.8 & $<0.001$ & 30.0 & $<0.001$ \\
\hline No & 53 & 84.2 & & 97.3 & & 74.2 & & 72.0 & & 81.0 & \\
\hline \multicolumn{12}{|l|}{ T stage } \\
\hline pT3-4 & 26 & 57.9 & $<0.001$ & 93.8 & 0.104 & 33.2 & $<0.001$ & 35.1 & $<0.001$ & 45.9 & $<0.001$ \\
\hline pT1-2 & 39 & 93.1 & & 96.7 & & 85.5 & & 82.2 & & 88.9 & \\
\hline \multicolumn{12}{|c|}{ Lymphovascular invasion } \\
\hline Yes & 15 & 51.0 & $<0.001$ & 90.0 & 0.569 & 29.6 & $<0.001$ & 30.5 & $<0.001$ & 44.4 & $<0.001$ \\
\hline No & 50 & 89.4 & & 96.9 & & 78.6 & & 75.0 & & 82.7 & \\
\hline \multicolumn{12}{|l|}{ Perineural invasion } \\
\hline Yes & 35 & 66.2 & 0.007 & 92.0 & 0.084 & 48.9 & 0.002 & 45.7 & 0.001 & 66.3 & 0.092 \\
\hline No & 30 & 95.7 & & 100 & & 87.9 & & 87.9 & & 78.0 & \\
\hline \multicolumn{12}{|l|}{ Margin $<1 \mathrm{~mm}$} \\
\hline Yes & 47 & 79.1 & 0.910 & 96.6 & 0.794 & 68.7 & 0.418 & 66.0 & 0.449 & 75.7 & 0.475 \\
\hline No & 18 & 80.9 & & 91.7 & & 57.0 & & 57.5 & & 61.1 & \\
\hline \multicolumn{12}{|l|}{ High-grade histology } \\
\hline Yes & 59 & 77.1 & 0.204 & 94.9 & 0.466 & 63.0 & 0.324 & 61.3 & 0.287 & 68.4 & 0.079 \\
\hline No & 6 & 100 & & 100 & & 85.7 & & 85.7 & & 100 & \\
\hline \multicolumn{12}{|l|}{ Multivariate analysis } \\
\hline Variable & & $\mathrm{HR}$ & $95 \% \mathrm{Cl}$ & & $P$ & Variable & & & $\mathrm{HR}$ & $95 \% \mathrm{Cl}$ & $P$ \\
\hline \multicolumn{6}{|l|}{ Regional control } & \multicolumn{6}{|c|}{ Disease-free survival } \\
\hline \multicolumn{2}{|c|}{ T stage (pT3-4 vs pT1-2) } & 8.129 & \multicolumn{2}{|c|}{$1.661-39.779$} & 0.010 & \multicolumn{3}{|c|}{ N stage (pN+ vs pNO \& cNO/pNxa) } & 4.026 & $1.464-11.075$ & 0.007 \\
\hline \multicolumn{2}{|c|}{ Lymphovascular invasion (Yes vs No) } & 4.130 & \multicolumn{2}{|c|}{$1.165-14.643$} & 0.028 & \multicolumn{3}{|c|}{ Perineural invasion (Yes vs No) } & 5.377 & $1.512-19.121$ & 0.009 \\
\hline \multicolumn{2}{|l|}{ Local control } & - & - & & NS & \multicolumn{3}{|c|}{ T stage (pT3-4 vs pT1-2) } & 3.762 & $1.262-11.213$ & 0.017 \\
\hline \multicolumn{6}{|c|}{ Distant metastasis-free survival } & \multicolumn{6}{|c|}{ Overall survival } \\
\hline \multicolumn{2}{|c|}{ T stage (pT3-4 vs pT1-2) } & 5.171 & $1.715-15$ & .591 & 0.004 & $\mathrm{~N}$ sta & $p N+v s p$ & $\left(\mathrm{cNO} / \mathrm{pNx}^{\mathrm{a}}\right)$ & 4.363 & $1.710-11.127$ & 0.002 \\
\hline Perineural invasio & No) & 4.337 & $1.234-15$ & .236 & 0.022 & T stas & $\mathrm{TT} 3-4$ vs & & 4.841 & $1.671-14.020$ & 0.004 \\
\hline Lymphovascular i & (Yes vs No) & 2.719 & $1.065-6$. & 942 & 0.037 & & & & & & \\
\hline
\end{tabular}

Abbreviations: Cl confidence interval, DFS disease free survival, DMFS distant metastasis free survival, HR Hazard ratio, LC local control, NS not statistically significant, $O S$ overall survival, $R C$ regional control

${ }^{a}$,no elective neck dissection in cNO patients

\section{Extended elective nodal irradiation}

To identify specific populations of SMGC patients who might benefit from extended ENI, we performed subgroup analyses to examine each pathological parameter. As demonstrated in Table 5 and Fig. 2a-d, extended ENI significantly improved $\mathrm{RC}$ rates for patients with $\mathrm{pN}+$ $(P=0.003)$, ENE $(P=0.022)$, pT3-4 $(P=0.044)$, and LVI $(P=0.014)$. For patients with $\mathrm{pN}+$, we observed a significant improvement in five-year DFS in those treated with extended ENI (52\% vs $0 \%, P=0.034$, Fig. 2e). Although higher five-year DMFS rates were observed for $\mathrm{pN}+$ patients treated with extended ENI (52\% vs $13 \%, P=0.166)$, as well as higher five- and 10-year OS rates (44 and 44\% vs 28 and $0 \%, P=0.333$ ), the differences were not significant.

To identify the subgroups of patients who might benefit the most from extended ENI, we stratified patients according to $\mathrm{pN}+$, ENE, LVI, and pT3-4 (the patient characteristics between the treatment groups are listed in Additional file 2: Table S1). In patients with $\geq 1$ adverse pathological factor(s), extended ENI to $\geq 1$ adjacent echelons significantly increased five- and 10-year RC rates ( 86 and $86 \%$ vs 45 and $45 \%$, respectively, $P=0.039$; 


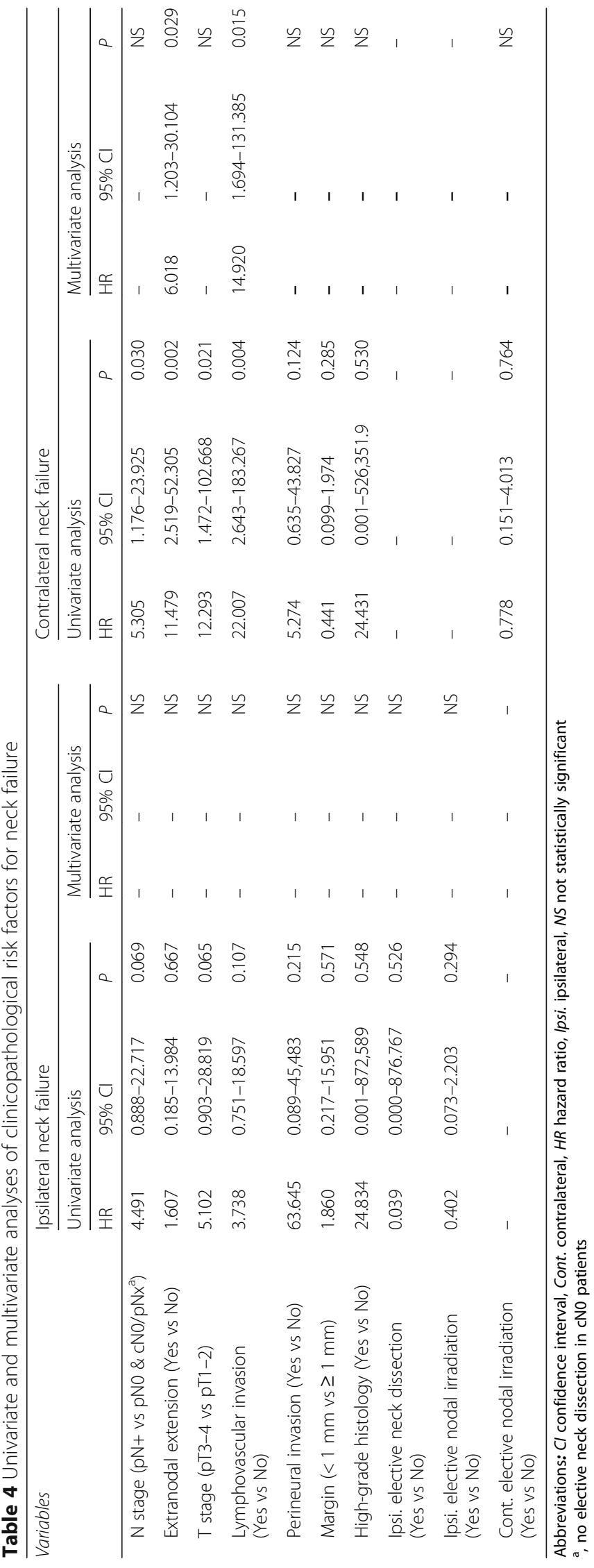


Table 5 Survival outcomes of submandibular gland carcinoma patients bearing adverse pathological risk factors, treatment with or without postoperative bilateral elective nodal irradiation

\begin{tabular}{|c|c|c|c|c|c|c|c|c|c|c|c|c|c|}
\hline \multirow[t]{2}{*}{ Variable } & \multirow[b]{2}{*}{$N$} & \multicolumn{3}{|c|}{$\mathrm{RC}(\%)$} & \multicolumn{3}{|c|}{ DMFS (\%) } & \multicolumn{3}{|c|}{ DFS (\%) } & \multicolumn{3}{|c|}{ OS (\%) } \\
\hline & & $5-y$ & $10-y$ & $P$ & $5-y$ & $10-y$ & $P$ & $5-y$ & $10-y$ & $P$ & $5-y$ & $10-y$ & $P$ \\
\hline \multicolumn{14}{|l|}{ Entire cohort } \\
\hline Extended ENI & 18 & 90.0 & 90.0 & 0.207 & 59.3 & 59.3 & 0.700 & 59.3 & 59.3 & 0.871 & 70.3 & 61.6 & 0.636 \\
\hline Limited ENI & 47 & 76.6 & 76.6 & & 67.6 & 67.6 & & 65.4 & 65.4 & & 72.3 & 63.6 & \\
\hline \multicolumn{14}{|l|}{$\mathrm{pN}+$} \\
\hline Extended ENI & 9 & 100 & 100 & 0.003 & 51.9 & 51.9 & 0.166 & 51.9 & 51.9 & 0.034 & 44.4 & 44.4 & 0.333 \\
\hline Limited ENI & 9 & 0 & - & & 12.5 & - & & 0 & - & & 27.8 & 0 & \\
\hline \multicolumn{14}{|c|}{ Extranodal extension } \\
\hline Extended ENI & 6 & 100 & 100 & 0.022 & 44.4 & 44.4 & 0.328 & 44.4 & 44.4 & 0.161 & 50.0 & 50.0 & 0.319 \\
\hline Limited ENI & 6 & 20.0 & - & & 0 & - & & 0 & - & & 0 & - & \\
\hline \multicolumn{14}{|l|}{ pT3-4 } \\
\hline Extended ENI & 8 & 100 & 100 & 0.044 & 40.0 & 40.0 & 0.694 & 40.0 & 40.0 & 0.579 & 37.5 & 18.8 & 0.288 \\
\hline Limited ENI & 18 & 41.0 & 41.0 & & 32.7 & 32.7 & & 34.3 & 34.3 & & 49.1 & 32.8 & \\
\hline \multicolumn{14}{|c|}{ Lymphovascular invasion } \\
\hline Extended ENI & 9 & 80.0 & 80.0 & 0.014 & 32.4 & 32.4 & 0.472 & 32.4 & 32.4 & 0.351 & 55.6 & 44.4 & 0.392 \\
\hline Limited ENI & 9 & 25.0 & 25.0 & & 25.0 & 25.0 & & 25.0 & 25.0 & & 33.3 & 22.2 & \\
\hline \multicolumn{14}{|c|}{ Perineural invasion } \\
\hline Extended ENI & 9 & 80.0 & - & 0.353 & 25.9 & - & 0.195 & 25.9 & - & 0.300 & 55.6 & 41.7 & 0.270 \\
\hline Limited ENI & 26 & 66.3 & 66.3 & & 57.0 & 57.0 & & 52.6 & 52.6 & & 69.4 & 52.9 & \\
\hline \multicolumn{14}{|l|}{ Margin $<1 \mathrm{~mm}$} \\
\hline Extended ENI & 12 & 83.3 & 83.3 & 0.512 & 61.1 & 61.1 & 0.905 & 61.1 & 61.1 & 0.763 & 71.4 & 53.6 & 0.374 \\
\hline Limited ENI & 35 & 78.0 & 78.0 & & 70.3 & 70.3 & & 66.8 & 66.8 & & 77.1 & 65.4 & \\
\hline \multicolumn{14}{|c|}{ High grade histology } \\
\hline Extended ENI & 41 & 88.9 & 88.9 & 0.179 & 63.3 & 63.3 & 0.899 & 63.3 & 63.3 & 0.650 & 68.4 & 58.6 & 0.721 \\
\hline Limited ENI & 17 & 73.1 & 73.1 & & 63.0 & 63.0 & & 60.4 & 60.4 & & 68.6 & 58.8 & \\
\hline \multicolumn{14}{|l|}{0 risk factor ${ }^{a}$} \\
\hline Extended ENI & 6 & 100 & 100 & - & 100 & 100 & 0.533 & 100 & 100 & 0.533 & 100 & 100 & 0.449 \\
\hline Limited ENI & 26 & 100 & 100 & & 91.1 & 91.1 & & 91.1 & 91.1 & & 87.9 & 87.9 & \\
\hline \multicolumn{14}{|l|}{$\geq 1$ risk factor ${ }^{a}$} \\
\hline Extended ENI & 12 & 85.7 & 85.7 & 0.039 & 43.2 & 43.2 & 0.558 & 43.2 & 43.2 & 0.349 & 58.3 & 48.6 & 0.752 \\
\hline Limited ENI & 21 & 45.1 & 45.1 & & 38.2 & 38.2 & & 31.5 & 31.5 & & 52.4 & 32.7 & \\
\hline \multicolumn{14}{|l|}{$\geq 2$ risk factors ${ }^{a}$} \\
\hline Extended ENI & 11 & 83.3 & 83.3 & $<0.001$ & 36.8 & 36.8 & 0.019 & 36.8 & 36.8 & 0.007 & 54.5 & 43.6 & 0.117 \\
\hline Limited ENI & 8 & 14.3 & - & & 0 & 0 & & 0 & 0 & & 16.7 & 0 & \\
\hline
\end{tabular}

Abbreviations: DFS disease free survival, DMFS distant metastasis free survival, ENI elective nodal irradiation, OS overall survival, RC regional control

${ }^{\mathrm{a}}$ Risk factors: $\mathrm{pN}+$, extranodal extension, $\mathrm{pT} 3-4$, and lymphovascular invasion

Table 5 and Fig. 2f). However, the DMFS, DFS, and OS rates did not significantly differ between each group. For patients with $\geq 2$ coincident adverse pathological factors, extended ENI to the adjacent echelons bilaterally increased five-year RC ( $83 \%$ vs $14 \%, P<0.001$, Fig. 2 g), DMFS (37\% vs $0 \%, P=0.019$, Fig. 2 h), and DFS (37\% vs $0 \%, P=0.007$, Fig. $2 \mathrm{i}$ ) rates significantly. However, the fiveand 10-year OS rates were not significantly higher in patients treated with extended ENI (55 and 44\% vs 16.7 and
$0 \%$, respectively; $P=0.117$, Fig. $2 \mathrm{j})$. On the other hand, the five- and 10 -year RC rates were $100 \%$ and $100 \%$ in patients who did not receive extended ENI $(N=26)$ but had none of the aforementioned adverse pathological factors; neither elective nodal dissection nor irradiation of the adjacent echelons was performed in 14 of these patients. Furthermore, no significant differences were observed in terms of RC, DMFS, DFS, and OS rates in these low-risk patients whether treated with extended ENI or not. 
a
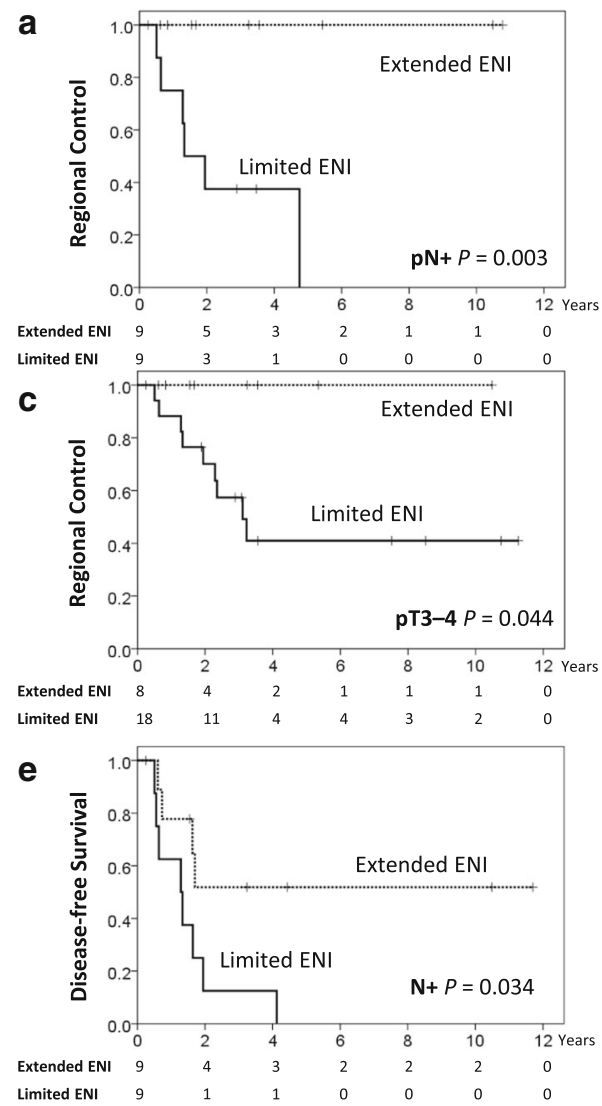

g

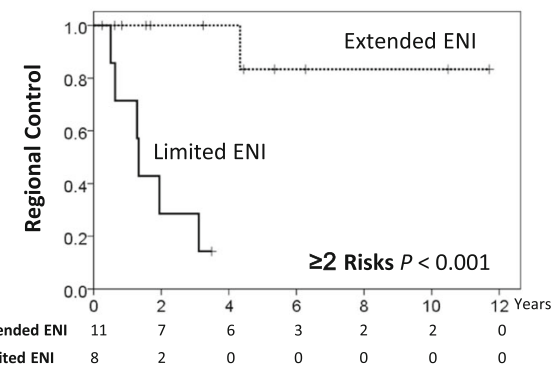

i

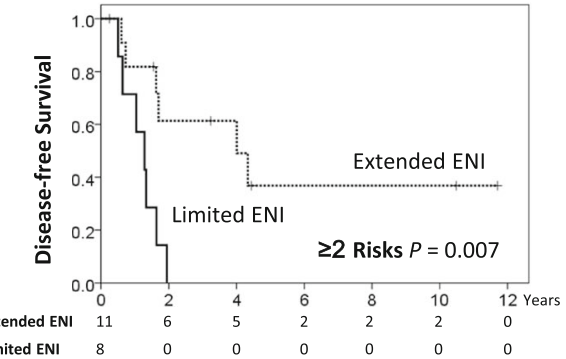

b

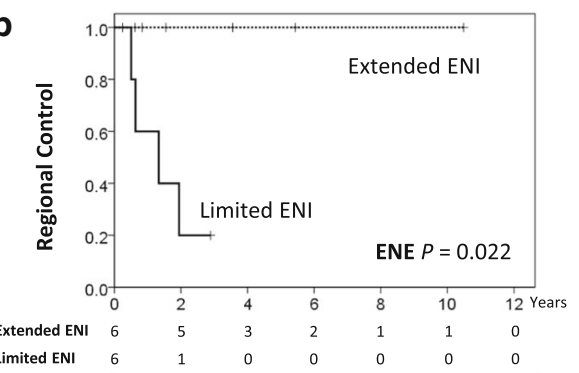

d

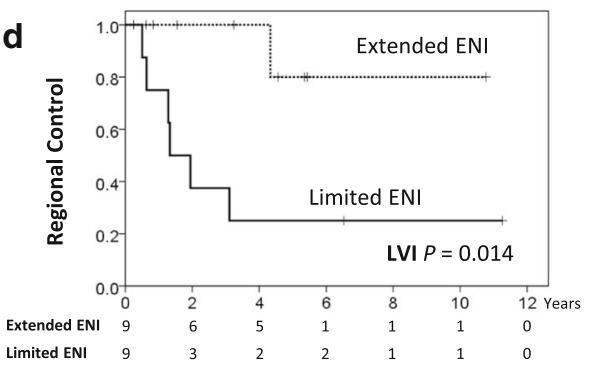

f

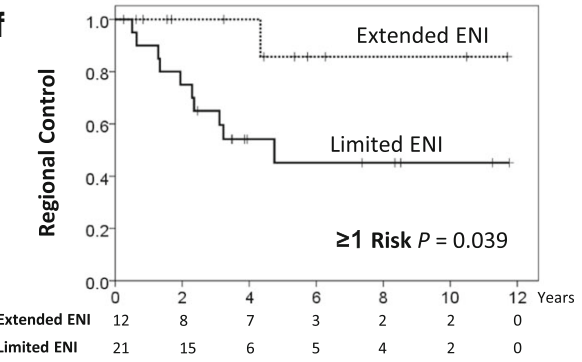

h

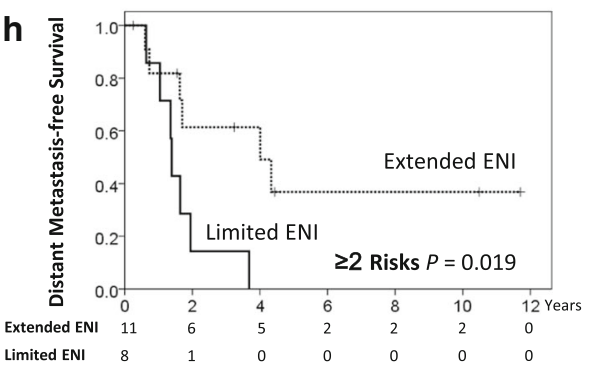

j

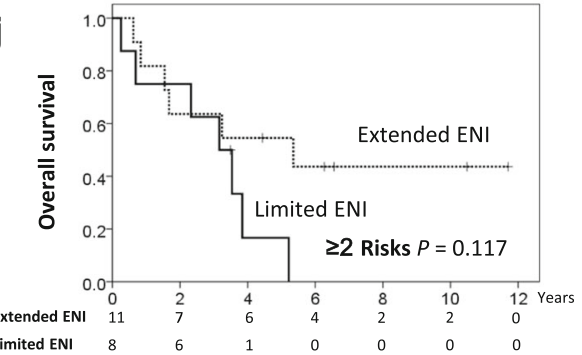

Fig. 2 a-d Regional control curves in patients with pN+, extranodal extension (ENE), pT3-4, and lymphovascular invasion (LVI); and (e) diseasefree survival curves in $\mathrm{pN}(+)$ patients; treated with extended vs limited elective nodal irradiation (ENI). $\mathbf{f}$ Regional control curves in patients with $\geq 1$ adverse factors, and $(\mathbf{g}-\mathbf{j})$ regional control, distant metastasis-free survival, disease-free survival, and overall survival curves in patients with $\geq 2$ adverse factors treated with extended or limited ENI. (Adverse factors: pN+, ENE, pT3-4, and LVI)

\section{Discussion}

Despite their generally indolent clinical course, nodal and distant recurrences remain a major clinical concern in SMGC patients who carry adverse pathological factors, even following combined-modality treatment $[1,2,6,8$, 26]. The recommended ENI fields of PORT for SMGC derive from the indications outlined for parotid cancer, where ipsilateral level I-V irradiation is adopted for patients with 
advance T-stage, $\mathrm{pN}(+)$, high-grade histology, PNI, and recurrent disease. Ipsilateral upper neck ENI is recommended for cases with early high-risk tumors [6, 10-13].

However, the submandibular gland has a rich lymphocapillary network. A large surgical series revealed that submandibular malignancies had a significantly higher risk of occult nodal metastasis (21\%) than parotid gland cancers (9\%) [10]. In our previous study of salivary gland cancer patients treated with PORT, those with SMGC exhibited a significantly lower five-year RC rate (73.9\%) compared to patients with tumors originating from other salivary glands (parotid, 91.2\%; sublingual, 100\%; and minor salivary, 100\%) [23]. These data suggest that the nodal spreading behavior of SMGCs differs from that of parotid gland tumors, and more aggressive treatment may be warranted for select high-risk SMGC patients.

In the present study, the overall incidence of neck metastasis was as high as $35 \%(\mathrm{pN}+, N=18$ [27\%]; neck recurrence in patients with $\mathrm{pNO}$ or $\mathrm{cN} 0 / \mathrm{pNx}$ [i.e., no elective neck dissection performed for clinically negative necks], $N=5$, [8\%]; Fig. 1d-f) which is consistent with other series [1, 17, 27-29]. Occult metastasis was documented in $9 \%$ of our patients (pN+ in cN0 patients, $N=1$; neck recurrence in $\mathrm{pN} 0$ or $\mathrm{cN} 0 / \mathrm{pNx}, N=5$ ). Our data indicated that contralateral nodal metastasis was not uncommon in SMGC patients (Fig. 1d), particularly in the presence of $\mathrm{pN}+$, ENE, pT3-4, and LVI (Table 4), suggesting that ipsilateral ENI of the neck may be inadequate for these high-risk subgroups. However, our data did not identify predictors for ipsilateral neck relapse, possibly owing to the small number of events and the fact that ipsilateral neck prophylactic treatment was frequently performed for patients with adverse pathological factors. Importantly, we found that $64 \%$ of nodal recurrences were located in the adjacent uninvolved echelons, suggesting that ENI of the adjoining echelons might be warranted in selected patients. Notably, 10 of the 11 patients who experienced nodal relapse (91\%) also developed distant metastases; this strong correlation implies that reducing nodal recurrence might in turn decrease the risk of distant failure.

Positive surgical margins are a poor prognostic factor and have been reported in 36-46\% of SMGC patients $[17,28]$. Compared to other surgical series, we observed a higher incidence of surgical margins < $1 \mathrm{~mm}$ (71\%). This might be attributed to more conservative resections as well as the clinical aggressiveness of SMGC. Intriguingly, our data demonstrated that resection margins $<1 \mathrm{~mm}$ were not significantly associated with poor outcomes in the setting of adjuvant radiotherapy; this was comparable to our previous findings with parotid cancer [30]. This may indicate the high efficacy of adjuvant radiotherapy in eradicating microscopic tumors, especially as only two patients experienced nodal relapses within the initial irradiation fields.

We hypothesized that the anatomic proximity of SMGCs to the floor of the mouth and midline of the neck increases the risk of contralateral neck and occult metastasis, and that ipsilateral or limited ENI is inadequate in such cases. Therefore, we categorized the ENI fields into three consecutive bilateral echelons accordingly to anatomical lymphatic drainage. We found no statistically significant differences in disease control and survival rates between patients treated with or without extended ENI. However, in subgroup analyses, extended ENI significantly improved RC rates in patients exhibiting adverse pathological factors. In $\mathrm{pN}+$ patients in particular, a significantly higher DFS rate was observed in those treated with extended ENI, suggesting that a reduced nodal failure rate might translate into a DFS benefit.

To identify SMGC patients who may benefit most from extended ENI, we stratified our patients according to the aforementioned adverse factors. For patients with $\geq 1$ risk factor(s), bilateral ENI extension to the adjacent echelons significantly improved the five-year $\mathrm{RC}$ rate; however, there were no significant differences in DMFS, DFS, and OS rates between the groups. Importantly, for patients with $\geq 2$ coincident risk factors, extended ENI significantly increased the five-year RC, DMFS, and DFS rates. The five- and 10-year OS rates were also (non-significantly) higher in patients treated with extended ENI, suggesting that patients bearing multiple coincident adverse factors may derive the most benefit from extended ENI. Conversely, for patients without any of the aforementioned risk factors, irradiation of the involved echelon alone appears to be sufficient for decreasing treatment-related morbidities.

Concordant with the published literature [17, 31], our multivariate analysis identified PNI as an independent predictor for worse DMFS and DFS, but not for inferior RC. Additionally, no significant RC improvement was observed in patients with perineural invasion treated with extended ENI, suggesting that the presence of PNI alone may not warrant the use of extended ENI. Nonetheless, the current study documented that 19 (66\%) out of 29 ACC patients presented with PNI, and two (11\%) of the 19 developed outfield perineural tumor recurrence. Since a higher propensity of perineural invasion/spread in ACC has been documented [32,33], prophylactic irradiation to the nerve tract should be strongly recommended particularly in the presence of PNI and locally-advanced disease. However, the distance from the tumor at which radiation treatment should be administered cannot be determined by this limited data. Coverage of the nerve tract of the lingual nerve, hypoglossal nerve, and facial nerve (marginal mandibular branch) to the skull base might be adequate in preventing morbidity as a result of brain irradiation. Additionally, it is warranted to extend outwards to cover the angle of the jaw and the 
plane between the plasma for the marginal mandibular branch.

We observed a high nodal recurrent rate (17\%) in SMGC patients treated with PORT. Additionally, failures at contralateral neck nodes and uninvolved adjacent echelons were frequently observed, particularly in patients with $\mathrm{pN}+$, ENE, pT3-4, and LVI. To our knowledge, we are the first to demonstrate the indications and clinical utility of extended ENI for the bilateral treatment of adjacent echelons of SMGC. However, there are some limitations inherent to this retrospective study. While ours was the largest long-term series of SMGC patients treated with PORT, the relatively small number of subjects may have biased our results. The median follow-up of 79 months was relatively short for accurately evaluating the survival outcomes for SMGC. Additionally, we cannot fully account for all potential biases due to the non-randomized retrospective nature of the study. The subjective treatment decisions and the diverse irradiation fields used may also be regarded as limitations. However, because of the low prevalence and indolent nature of salivary gland malignancies, conducting a decade-long prospective randomized trial would be difficult. The only ongoing randomized trial, RTOG 1008, is designed to investigate the efficacy of postoperative cisplatin-based chemoradiation in patients with salivary gland carcinomas; the target enrollment size is 120 patients. Importantly, this trial does not specifically assess SMGC patients; the neck irradiation field for SMGC is based on empirical experience. Hence, we believe that our multicenter experience is valuable and provides a rationale for the design of future prospective trials.

\section{Conclusion}

Our long-term study showed that nodal recurrence on the contralateral side of the neck and adjacent uninvolved echelons was not uncommon in SMGC patients treated with PORT, and that this should be considered during radiotherapy planning. Extended ENI appears to improve RC and DFS rates in patients with certain (or multiple) adverse pathological factors. Conversely, limited ENI to the involved echelons alone appears to be adequate for low-risk patients without risk factors.

\section{Additional files}

Additional file 1: Figure S1. Perineural tumor recurrence at (A) lingua (solid arrow), inferior alveolar (arrow head) and facial (dashed arrow) nerves (\#12) and (B) hypoglossal nerve (\#11). (PDF 63 kb)

Additional file 2: Table S1. Patient characteristics in selected patients with risk factors. (DOCX 334 kb)

\section{Acknowledgements}

We are grateful to the members of the Head and Neck Oncology Group at Linkou Chang Gung Memorial Hospital for their invaluable help.

\section{Funding}

This study was financially supported by Research Grants CMRPG3F0061, CMRPG3F0061-2, CMRPG3F0861 and CORPG3G0961 from the Chang Gung Medical Foundation

\section{Availability of data and materials}

The datasets used during the present study are available from the corresponding author on reasonable request.

\section{Honors}

Portions of these data were presented at the ASTRO's 56th Annual Meeting (2014; San Francisco, CA, USA). This study was awarded the Outstanding Research Prize by the Chinese Oncology Society in 2014.

\section{Authors' contributions}

CEH and CYL conceived of the study. CEH and CYL carried out the manuscript writing and revision for important intellectual content. LYL and CEH performed histology specimen collection and review. CEH and CYL carried out the statistical analyses. KHF, CTL, JTCC, NMT, HMW, KHF, SHN and TCY participated in data collection and patient clinical care. All authors have read and approved the final manuscript.

Ethics approval and consent to participate

This study was approved by the Chang Gung Medical Fundation Institutional Review Boards (IRB; 102-0938B and 104-2363B).

Consent for publication

Not applicable.

Competing interests

The authors declare that they have no competing interests.

\section{Publisher's Note}

Springer Nature remains neutral with regard to jurisdictional claims in published maps and institutional affiliations.

\section{Author details}

${ }^{1}$ Radiation Oncology, Chang Gung Memorial Hospital at Linkou and Chang Gung University, No. 5, Fuxing St., Guishan Dist, Taoyuan City 33305, Taiwan, Republic of China. ${ }^{2}$ Pathology, Chang Gung Memorial Hospital at Linkou and Chang Gung University, Taoyuan City, Taiwan, Republic of China. ${ }^{3}$ Medical Oncology, Chang Gung Memorial Hospital at Linkou and Chang Gung University, Taoyuan City, Taiwan, Republic of China. ${ }^{4}$ Diagnostic Radiology, Chang Gung Memorial Hospital at Linkou and Chang Gung University, Taoyuan City, Taiwan, Republic of China. ${ }^{5}$ Otorhinolaryngology, Head and Neck Surgery, Chang Gung Memorial Hospital at Linkou and Chang Gung University, Taoyuan City, Taiwan, Republic of China. ${ }^{6}$ Nuclear Medicine and Molecular Imaging Center, Chang Gung Memorial Hospital at Linkou and Chang Gung University, Taoyuan City, Taiwan, Republic of China. ${ }^{7}$ Head and Neck Oncology Group, Chang Gung Memorial Hospital at Linkou and Chang Gung University, Taoyuan City, Taiwan, Republic of China. ${ }^{8}$ Graduate Institute of Clinical Medical Science, Chang Gung University, Taoyuan City, Taiwan, Republic of China. ${ }^{9}$ School of Traditional Chinese Medicine, Chang Gung University, Taoyuan City, Taiwan, Republic of China. ${ }^{10}$ Radiation Oncology, Xiamen Chang Gung Hospital, Xiamen City, Fujian Province, China.

${ }^{11}$ Departments of Experimental Radiation Oncology, Division of Radiation Oncology, The University of Texas MD Anderson Cancer Center, Houston, Texas, USA. ${ }^{12}$ The University of Texas MD Anderson Cancer Center-UT Health Graduate School of Biomedical Sciences, Houston, Texas, USA. ${ }^{13}$ Particle Physics and Beam Delivery Core Laboratory, Institute for Radiological Research, Chang Gung Memorial Hospital at Linkou and Chang Gung University, Taoyuan City, Taiwan, Republic of China.

Received: 8 September 2017 Accepted: 12 September 2018

Published online: 21 September 2018

\section{References}

1. Bhattacharyya N. Survival and prognosis for cancer of the submandibular gland. J Oral and Maxillofac Surg. 2004;62:427-30. 
2. Wahlberg P, Anderson H, Biörklund A, et al. Carcinoma of the parotid and submandibular glands - a study of survival in 2465 patients. Oral Oncol. 2002;38:706-13.

3. Spiro RH. Salivary neoplasms: overview of a 35-year experience with 2,807 patients. Head Neck Surg. 1986:8:177-84.

4. Adelstein DJ, Koyfman SA, El-Naggar AK, Hanna EY. Biology and Management of Salivary Gland Cancers. Semin Radiat Oncol. 2012;22:245-53.

5. Mendenhall WM, Morris CG, Amdur RJ, et al. Radiotherapy alone or combined with surgery for salivary gland carcinoma. Cancer. 2005;103:2544-50.

6. Terhaard $\mathrm{CH}$, Lubsen $\mathrm{H}$, Rasch $\mathrm{CR}$, et al. The role of radiotherapy in the treatment of malignant salivary gland tumors. Int J Radiat Oncol Biol Phys. 2005;61:103-11.

7. Zeidan $Y H$, Pekelis $L, A n Y$, et al. Survival benefit for adjuvant radiation therapy in minor salivary gland cancers. Oral Oncol. 2015;51:438-45.

8. Terhaard $\mathrm{CH}$, Lubsen $\mathrm{H}$, Van der Tweel I, et al. Salivary gland carcinoma: independent prognostic factors for locoregional control, distant metastases, and overall survival: results of the Dutch head and neck oncology cooperative group. Head Neck. 2004;26:681-93.

9. Armstrong JG, Harrison LB, Spiro RH, et al. Malignant tumors of major salivary gland origin. A matched-pair analysis of the role of combined surgery and postoperative radiotherapy. Arch Otolaryngol Head Neck Surg. 1990;116:290-3

10. Armstrong JG, Harrison $L B$, Thaler $H T$, et al. The indications for elective treatment of the neck in cancer of the major salivary glands. Cancer. 1992;69:615-9.

11. Ferlito A, Pellitteri PK, Robbins KT, et al. Management of the neck in cancer of the major salivary glands, thyroid and parathyroid glands. Acta Otolaryngol. 2002;122:673-8.

12. Chen AM, Garcia J, Lee NY, et al. Patterns of nodal relapse after surgery and postoperative radiation therapy for carcinomas of the major and minor salivary glands: what is the role of elective neck irradiation? Int J Radiat Oncol Biol Phys. 2007;67:988-94.

13. Chen AM, Granchi PJ, Garcia J, et al. Local-regional recurrence after surgery without postoperative irradiation for carcinomas of the major salivary glands: implications for adjuvant therapy. Int J Radiat Oncol Biol Phys. 2007; 67:982-7.

14. Zbären $P$, Schüpbach J, Nuyens M, Stauffer E. Elective neck dissection versus observation in primary parotid carcinoma. Otolaryngol Head Neck Surg. 2005:132:387-91.

15. Vander Poorten VL, Balm AJ, Hilgers FJ, et al. The development of a prognostic score for patients with parotid carcinoma. Cancer. 1999;85:2057-67.

16. Régis De Brito Santos I, Kowalski LP, Cavalcante De Araujo V, et al. Multivariate analysis of risk factors for neck metastases in surgically treated parotid carcinomas. Arch Otolaryngol Head Neck Surg. 2001;127:56-60.

17. Han MW, Cho K-J, Roh J-L, et al. Patterns of lymph node metastasis and their influence on outcomes in patients with submandibular gland carcinoma. J Surg Oncol. 2012;106:475-80.

18. Group IHaNS. Cervical lymph node metastasis in adenoid cystic carcinoma of the major salivary glands. J Laryngol Otol. 2017;131:96-105.

19. Lin T-C, Tsou Y-A, Bau D-T, Tsai M-H. Factors influencing contralateral neck metastasis in oral squamous cell carcinoma. Formos J Surg. 2012;45:83-7.

20. Dias FL, Lima RA, Kligerman J, et al. Relevance of skip metastases for squamous cell carcinoma of the oral tongue and the floor of the mouth. Otolaryngol Head Neck Surg. 2006;134:460-5.

21. Pimenta Amaral TM, da Silva Freire AR, Carvalho $A L$, et al. Predictive factors of occult metastasis and prognosis of clinical stages I and II squamous cell carcinoma of the tongue and floor of the mouth. Oral Oncol. 2004;40:780-6.

22. Liao C-T, Chang J-C, Wang H-M, et al. Analysis of risk factors of predictive local tumor control in oral cavity cancer. Ann Surg Oncol. 2008;15:915-22.

23. Hsieh C-E, Lin C-Y, Lee L-Y, et al. Postoperative radiotherapy with or without concurrent chemotherapy for salivary gland carcinomas. Therapeut Radiol Oncol. 2015;22:89-91.

24. Tsan D-L, Lin C-Y, Kang C-J, et al. The comparison between weekly and three-weekly cisplatin delivered concurrently with radiotherapy for patients with postoperative high-risk squamous cell carcinoma of the oral cavity. Radiat Oncol. 2012;7:215.

25. Hsieh C-E, Lin C-Y, Lee L-Y, et al. Adding concurrent chemotherapy to postoperative radiotherapy improves locoregional control but not overall survival in patients with salivary gland adenoid cystic carcinoma-a propensity score matched study. Radiat Oncol. 2016;11:1-10.

26. Johnston ML, Huang SH, Waldron JN, et al. Salivary duct carcinoma: treatment, outcomes, and patterns of failure. Head Neck. 2016;38:E820-6.
27. Stennert $E$, Kisner D, Jungehuelsing M, et al. High incidence of lymph node metastasis in major salivary gland cancer. Arch Otolaryngol Head Neck Surg. 2003;129:720-3.

28. Mallik S, Agarwal J, Gupta T, et al. Prognostic factors and outcome analysis of submandibular gland cancer: a clinical audit. J Oral Maxillofac Surg. 2010; 68:2104-10.

29. Storey MR, Garden AS, Morrison WH, et al. Postoperative radiotherapy for malignant tumors of the submandibular gland. Int J Radiat Oncol Biol Phys. 2001:51:952-8.

30. Huang B-S, Chen W-Y, Hsieh C-E, et al. Outcomes and prognostic factors for surgery followed by modern radiation therapy in parotid gland carcinomas. Jpn J Clin Oncol. 2016;46:832-8.

31. Bjorndal K, Krogdahl A, Therkildsen $\mathbf{M H}$, et al. Salivary gland carcinoma in Denmark 1990-2005: a national study of incidence, site and histology. Results of the Danish head and neck Cancer group (DAHANCA). Oral Oncol. 2011;47:677-82.

32. Barrett AW, Speight PM. Perineural invasion in adenoid cystic carcinoma of the salivary glands: a valid prognostic indicator? Oral Oncol. 2009;45:936-40.

33. Chen AM, Bucci MK, Weinberg V, et al. Adenoid cystic carcinoma of the head and neck treated by surgery with or without postoperative radiation therapy: prognostic features of recurrence. Int J Radiat Oncol Biol Phys. 2006;66:152-9.
Ready to submit your research? Choose BMC and benefit from:

- fast, convenient online submission

- thorough peer review by experienced researchers in your field

- rapid publication on acceptance

- support for research data, including large and complex data types

- gold Open Access which fosters wider collaboration and increased citations

- maximum visibility for your research: over $100 \mathrm{M}$ website views per year

At $\mathrm{BMC}$, research is always in progress.

Learn more biomedcentral.com/submissions 